\title{
82. STATISTICS OF THE ORBITS OF METEOR STREAMS AND COMETS
}

\author{
V. N. LEBEDINETS, V. N. KORPUSOV, and A. K. SOSNOVA \\ Institute for Experimental Meteorology, Obninsk, U.S.S.R.
}

\begin{abstract}
From radar observations carried out during 1967-1968 a catalogue of 20000 orbits was obtained. A method has been developed for identifying meteor streams and associations, and the orbits of the meteors recorded during January-April 1968 have been analysed. Among them 163 streams have been identified, and these contain $33.5 \%$ of the total number of orbits. Comparison with the orbits of the short-period comets of $q<1.1$ AU shows considerable differences.
\end{abstract}

Radar measurements of the radiants and velocities of individual meteors were carried out at the Institute for Experimental Meteorology from September 1967 to August 1968 using the method described by Korpusov and Lebedinets (1970). The basic radar parameters were: wavelength $11.9 \mathrm{~m}$, transmitter pulse power $75 \mathrm{~kW}$, pulse repetition frequency $20 \mu \mathrm{s}$, transmitting and receiving antenna gain 16, maximum sensitivity of the basic receiver $0.5 \times 10^{-14} \mathrm{~W}$. The meteors recorded were mainly between magnitudes 5 and 8 . As a result of these measurements the orbits of 20000 meteoroids were calculated.

One of the main purposes of this work was the study of the orbits of meteor streams and associations. We developed a special method for the computer selection of meteor orbits in order to reveal the existence of meteor streams. In this method a meteor orbit is represented as a point in four-dimensional space $1 / a, e, i, \omega$. The orbits of sporadic meteoroids form a continuous background in the portion of this space satisfying the condition of collision with the Earth; inside a sufficiently small volume of the space the distribution of the sporadic background can be considered as accidental. Groups of orbits of meteoroids that are members of meteor streams are superimposed on the sporadic background.

The accidental $(\mathrm{O}-\mathrm{C})$ deviations of the radiants of individual meteors from the mean radiants for a number of known streams were evaluated according to the results of photographic observations (Whipple and Wright, 1954); see Table I. The (O-C) values are proportional to the stream-width and are several times less than the mean

TABLE I

$(\mathrm{O}-\mathrm{C})$ deviations of radiants of individual meteors from mean radiants for known streams

\begin{tabular}{llll}
\hline Stream & $\mathrm{O}-\mathrm{C}$ & Stream & $\mathrm{O}-\mathrm{C}$ \\
\hline Draconids & 0.12 & Southern Taurids & 0.57 \\
Leonids & 0.22 & Perseids & 0.68 \\
Geminids & 0.22 & Northern Taurids & 1.05 \\
Orionids & 0.38 & $\delta$ Aquarids & 0.80
\end{tabular}


square errors of the radar measurements of the radiants of individual stream meteors (Korpusov and Lebedinets, 1970). Consequently, we can consider as a first approximation that the spread of individual radar orbits is mainly due to measurement errors. It will be necessary eventually to take into account that, at least for some streams, the real spread of orbits of individual meteoroids can be compared with the spread due to measurement errors.

Mean square errors of the measurements of the coordinates of radiants $\left(\sigma_{\alpha}, \sigma_{\delta}\right)$ and atmosphere-free velocities $\left(\sigma_{v}\right)$ were determined by two methods: (1) by analysis of the different sources of error, and (2) according to the spread of radiants and velocities of individual meteors in well-known major meteor streams (Quadrantids, Geminids, Orionids, etc.).

The $\sigma_{\alpha}, \sigma_{\delta}$, and $\sigma_{v}$ values turned out to be dependent on the velocity and radiant position of a meteor and were expressed analytically as functions of $\alpha, \delta$, and $v$. The time of appearance of a meteor is determined with practically zero error. For each meteor mean square errors $\sigma_{1 / a}, \sigma_{e}, \sigma_{i}$, and $\sigma_{\omega}$ of the determination of the orbital elements were calculated from the values of $\sigma_{\alpha}, \sigma_{\delta}$, and $\sigma_{v}$.

There were two stages to the selection of meteor-stream orbits for each period of observation, the duration of which was 5 to 10 days. In the first stage meteor associations were outlined. A meteor was assumed to be related to a given association if the following conditions were satisfied:

$$
\begin{aligned}
& |(1 / a)-\overline{(1 / a)}|<2 \sigma_{1 / a}, \quad|i-\bar{i}|<2 \sigma_{i} \\
& |e-\bar{e}|<2 \sigma_{e}, \quad|\omega-\bar{\omega}|<2 \sigma_{\omega} .
\end{aligned}
$$

Here, $1 / a, e, i, \omega$ are the elements of the meteor orbit and $\overline{(1 / a)}, \bar{e}, \bar{i}, \bar{\omega}$ are the mean elements of the association orbit, calculated by successive approximations on the electronic computer in the process of search for the association. At the same time a region around the association was outlined, and meteors were assumed to be related if their orbits did not deviate from the mean orbit by more than $4 \sigma$. The main purpose of the first stage was to decrease the volume of information necessary for the final selection of meteor orbits.

In the second stage the membership of the associations and the mean orbits of the associations were obtained, and the probability was calculated that an association is merely an accidental fluctuation in the sporadic background. The mean orbit of $N_{K}$ association meteors confined by the sphere of radius $r_{K}$ in the four-dimensional space was calculated by successive approximations. This mean orbit is assumed to be the centre of the sphere, and the radius of the point characterizing the orbit of each meteor is calculated as

$$
r=\left\{\left[\frac{(1 / a)-\overline{(1 / a)}}{2 \sigma_{1 / a}}\right]^{2}+\left[\frac{e-\bar{e}}{2 \sigma_{e}}\right]^{2}+\left[\frac{i-\bar{i}}{2 \sigma_{i}}\right]^{2}+\left[\frac{\omega-\bar{\omega}}{2 \sigma_{\omega}}\right]^{2}\right\}^{1 / 2} .
$$

For each association $K$ mean orbits were calculated, their radii from $r_{K_{\min }}=0.2(K=1)$ to $r_{K_{\max }}=2.0(K=10)$.

The mean number of background meteors contained inside the sphere $N_{f K}=n_{f} v_{K}$ is calculated for each sphere, $v_{k}$ being the volume of the sphere of radius $r_{K}$ and $n$, the 
mean space density of background meteors in the vicinity of the association. This mean space density is taken to be the difference between the number of meteors corresponding to $K=7$ and $K=10$, divided by the difference between the volumes of these spheres, i.e.,

$$
n_{f}=\frac{N(K=10)-N(K=7)}{v(K=10)-v(K=7)} .
$$

If the sphere of radius $r_{K}$ is partially outside the region satisfying the conditions of collision with the Earth (these conditions being $1+e \geqslant 1 / a \geqslant 1-e$ for elliptical orbits and $1 / a \leqslant 1-e$ for hyperbolic orbits), then $v_{K}$ is equal to the volume of a truncated sphere.

The probability that the association is an accidental fluctuation in the background is found from the values of $N_{K}$ and $N_{f K}$ as

$$
p\left(N_{K}\right)=\sum_{i=0}^{2} \frac{\exp \left(-N_{f K}\right)\left(N_{f K}\right)^{N_{K}}}{N_{K_{i}} !}
$$

The most probable radius of the sphere confining the association meteors is chosen according to $p\left(N_{K}\right)$. For the majority of the streams the radius was found to be in the range 0.8 to 1.2 .

If the most probable radius of the sphere cannot be chosen according to the character of the variation of $p\left(N_{K}\right)$, it is assumed to be 0.8 . The mean orbit of the meteors inside the sphere of most probable radius for the association is assumed to be the orbit of the association including $N_{K}$ meteors, of which $N_{f K}$ can be background meteors. Further, we consider only the associations with $N_{K} \geqslant 5$.

The mean values of the radiant coordinates, velocities, and a number of other quantities characterizing the association, as well as the diurnal variations and mean square errors of these values, were then calculated.

By means of this procedure, the catalogue of 3970 meteor orbits recorded during the period January-April 1968 was analysed, and 163 streams (comprising 1330 orbits, or $33.5 \%$ of the whole) were identified.

An analogous investigation of radar meteor streams was carried out by Kashcheev et al. (1967), using the results of observations at Kharkov in 1960; however, the Kharkov catalogue contains no data for the period January to March, and our results therefore make up for this deficiency.

Comparison of our catalogue of orbits of meteor streams with the catalogue of minor streams by Terent'eva $(1963,1967)$ and with a portion of the Kharkov catalogue enabled us to identify 36 streams, 20 of which are identified with some confidence.

Of the 163 streams selected 161 have revolution periods $P<200$ yr. Thus, if we assume that meteoroids are the products of cometary disintegration, then for the majority of the streams we should look for a connection with short-period comets. Comparison of the stream orbits with cometary orbits enabled us to establish such a connection for only ten streams, the orbits of which are shown in Table II (which also includes two questionable cases). The coordinates of the cometary radiants, velocity, and orbital elements, the solar longitude $\lambda_{\odot}$ at the time of closest approach of the 
Earth to the cometary orbit, and the distance $\Delta$ between the Earth and the cometary orbit at that time are taken from Kramer's (1953) catalogue. For the majority of the streams the parent comets are not known.

The distributions of the orbital elements of the radar meteor streams and the shortperiod comets (Porter, 1961; Vsekhsvyatskij, 1967) are compared in Figures 1 to 3.
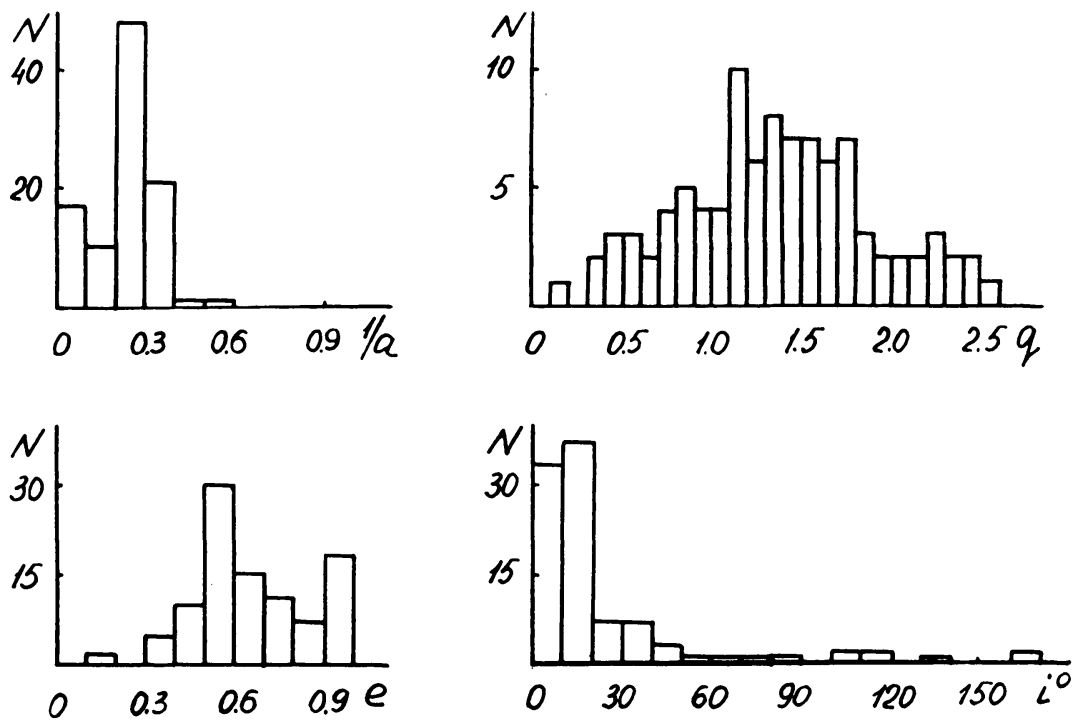

Fig. 1. Distribution of orbital elements of short-period comets.
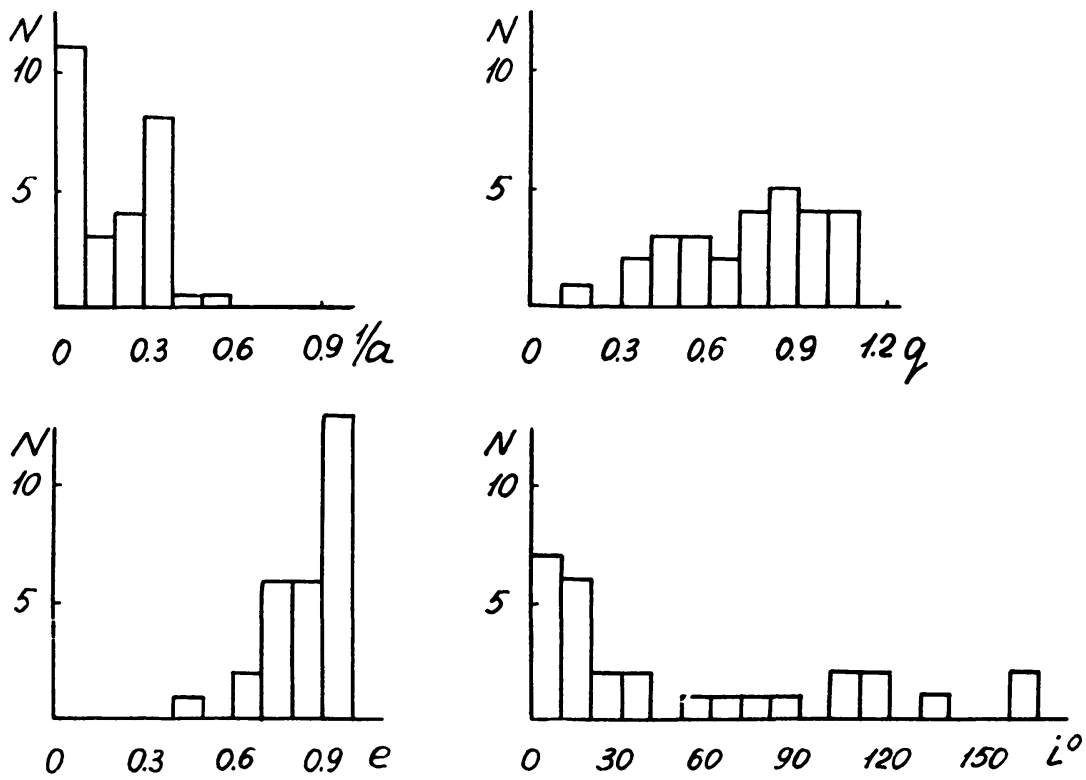

Fig. 2. Distribution of orbital elements of short-period comets with $q<1.1 \mathrm{AU}$. 
Figure 1 shows the distribution for all the short-period comets, while Figure 2 refers only to those with $q<1.1$ AU. The orbital distribution for the meteors is in Figure 3. The agreement of the $q$-distributions is good, the essential difference being that there are many streams of very small perihelion distance $(q<0.3 \mathrm{AU})$, but only one comet (and it has an orbit with $a=27.6 \mathrm{AU}$ ). In the case of the distributions in $1 / a$ and $e$ the differences are essentially greater.
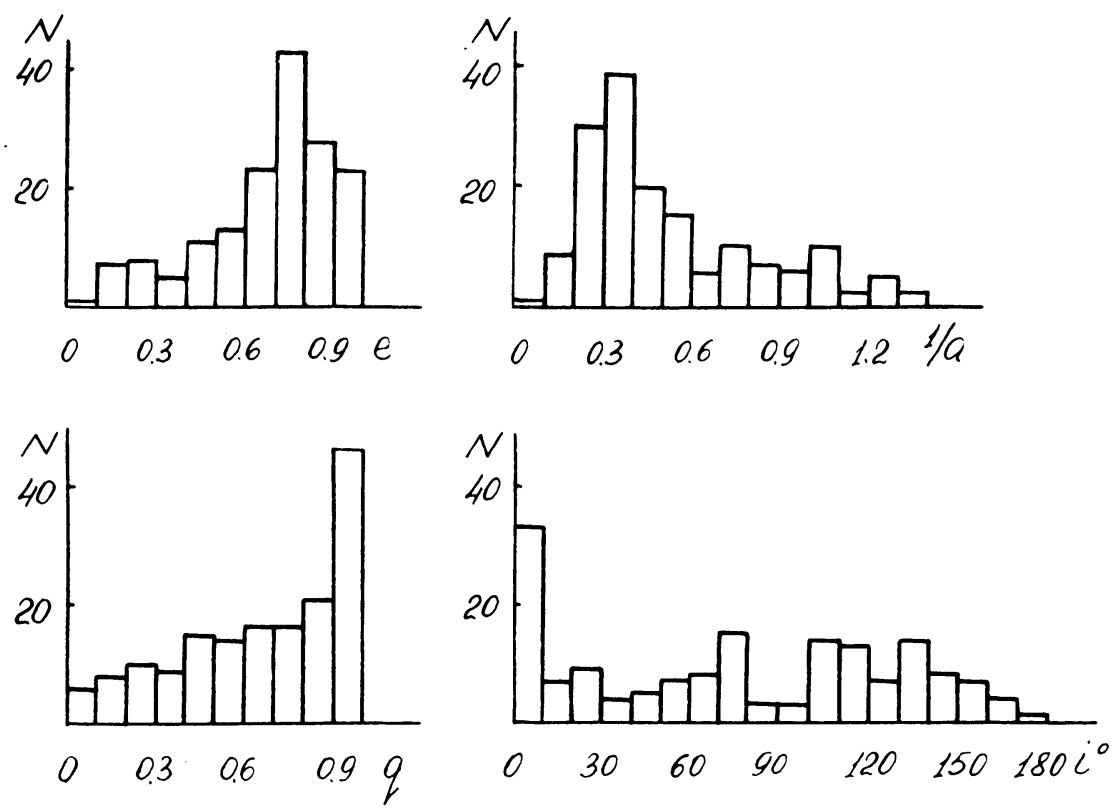

Fig. 3. Distribution of orbital elements of radar meteor streams.

Comparing the distributions of orbital elements of the meteor streams and shortperiod comet orbits over the four elements $1 / a, e, q$, and $i$, we deduce:

(1) Some of the observed meteor streams might have been generated as the result of distintegration of short-period comets;

(2) There is an essential difference between the orbits that cannot be explained solely by the evolution of the meteor orbits; this difference probably indicates that the character of the orbits of a considerable proportion of the meteor streams already differed considerably from that of the orbits of the known short-period comets at the time the streams first appeared.

The total observed complex of meteor streams with $P<200 \mathrm{yr}$ could not have been generated as the result of the disintegration of comets moving in orbits similar to those of the known short-period comets.

\section{References}

Kashcheev, B. L., Lebedinets, V. N.. and Lagutin, M. F.: 1967, Meteornye Yarleniya v Atmosfere Zemli, Nauka, Moscow. 
Korpusov, V. N. and Lebedinets, V. N.: 1970, Astron. Vestn. 4, 102.

Kramer, E. N.: 1953, Izv. Astron. Obs. Odess. Gos. Univ. 3, 163.

Porter, J. G.: 1961, Mem. Br. Astron. Assoc. 39, No. 3.

Terent'eva, A. K.: 1963, Astron. Tsirk. Nos. 249, 264.

Terent'eva, A. K.: 1967, Astron. Tsirk. Nos. 415, 423.

Vsekhsvyatskij, S. K.: 1967, Komety 1961-1965, Moscow.

Whipple, F. L. and Wright, F. W.: 1954, Monthly Notices Roy. Astron. Soc. 114, 229. 